\title{
Identification of mineral particles in pneumoconiotic lungs
}

W. J. HENDERSON, J. GOUGH, AND J. HARSE

From the Tenovus Institute for Cancer Research and the Department of Pathology, Welsh National School of Medicine, Cardiff

SYNOPSIS An extraction replication technique has been used for the study, by electron micr $\vec{\nabla}$ scopy, of lung tissue from a number of different cases of pneumoconiosis.

The technique provides a relatively simple means of studying the surface area of replicated tissues, and any foreign particles present which are either replicated or extracted from the tissue can be identified. A set of standard micrographs of different types of mineral particles likely to be encountered should be kept for reference. Most of the particles identified by th technique were beyond the limits of resolution of optical microscopy. Furthermore, the procedure involves the minimum of chemical and physical treatment.

The histochemical diagnosis and industrial history was confirmed in the first two case described, by the electron microscopy investigations. The third case presented an uncertai histological and industrial history, but an electron microscopy study confirmed the presence of the mineral particle considered responsible for the clinical condition. The fourth and fift cases described illustrate the use of this technique in identifying a particular type of pleomo phic mineral, and illustrating its position in situ in relation to surrounding tissue.

The accurate identification of the mineral particles considered responsible for the development of the pneumoconiotic condition in individuals working in certain industries has always been difficult. Histological studies on lung tissue from such patients can often be misleading, since most of the fine particles embedded in the lung tissue are beyond the resolution limits of the optical microscope. Chemical analysis of the material derived from ashed lung samples can also provide misleading interpretation due to chemical changes brought about by incineration. Many particles also change their shape and often disintegrate during the incineration process.

The procedure now described, involving an extraction-replication technique for the study of pneumoconiotic lung tissue by electron microscopy, provides the minimum of chemical and physical ill treatment. It enables fine particles to be identified, and the replication procedure presents an electron micrograph illustrating the Received for publication 13 June 1969. position of the particle in situ, in relation to the surrounding tissue.

\section{Materials and Methods}

\section{TISSUES}

The lung tissue studied was obtained from five patients with pneumoconiosis. The tissue had been prepared as a paraffin section for normat routine histological examination, but was un? stained.

MICROSCOPE AND REPLICATION TECHNIQU The procedure used was that described b\$ Henderson (1969). Paraffin wax was removed from the sections by immersion in xylene, and the tissue dehydrated in ethanol. The dehydrates tissue was embedded by impressing the sectio on to the surface of a sheet of acetone-softenee cellulose acetate $(0.5 \mathrm{~mm}$ thick, $25 \times 25 \mathrm{~mm}$ 
(a)

Cellulose acetate

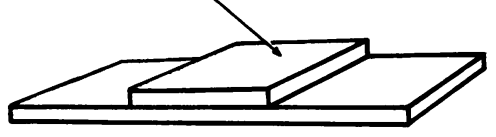

(b)

Histological thin section on slide

(C)

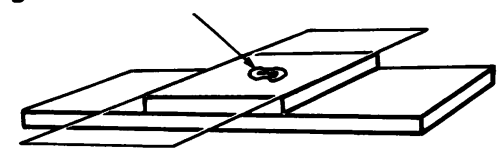

Scotch tape

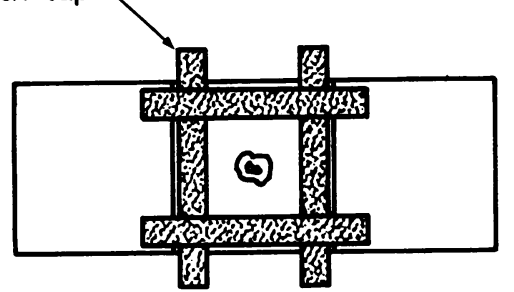

(d)

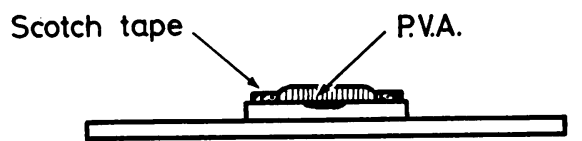

(e)

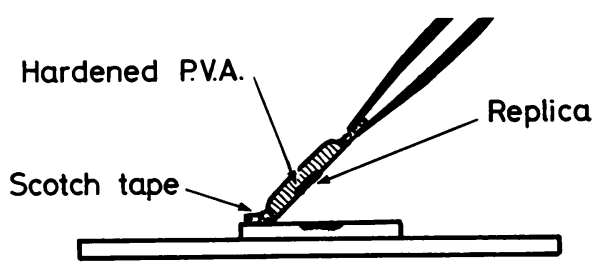

Fig. 1 Diagram of replication technique.

square mounted on a glass slide for easy handling) (Fig. 1a) which was then left to harden (Figure 1b). The slide was removed, leaving behind most of the tissue embedded in the cellulose acetate. The tissue was then outlined with strips of Scotch tape (Fig. 1c) to form a shallow well, and a $10 \%$ $(\mathrm{v} / \mathrm{v})$ solution of polyvinyl alcohol (PVA) was applied to the tissue (Figure 1d). Once the PVA hardened, it was stripped from the section, providing a replica of the tissue surface (Figure 1e). During this stripping process embedded particles are often removed with the PVA. This procedure allows successive strippings to be taken and a complete sequential examination through the embedded tissue. Surface replicas were preshadowed with platinum, and a carbon film deposited, the PVA was then removed by floating the replica in a hot water bath. The replicas were then mounted on electron microscope grids for examination. The AEI-6B electron microscope was used for all the studies described.
Results

The first patient, aged 67 at the time of death, presented with a productive cough and dyspnoea for some 10 years before his first medicalo examination. His industrial history was signifi cant, having worked in the china clay industry, bagging kaolin for more than 30 years. Radiology? showed massive fibrosis, and the necropsy음. revealed silicotic lungs, with the histology pre- $\frac{\bar{s}}{\frac{1}{}}$ senting a classical picture of kaoliniosis. Electron $\mathbb{\complement}^{\circ}$ microscope studies of the tissue replicas showed $ळ$ the presence of typical kaolinite particles associated with the lung tissue (Figure 2).

In the second case a patient aged 54 presented $\vec{\omega}$ with the usual symptoms of cough and dyspnoea. An industrial history of exposure to Norwegiano talc over the last 20 years of his industrial life wasi obtained. At necropsy, the pathologist considered $i$ that the lungs exhibited the microscopic appear- $\overrightarrow{0}$ ance of silicosis, whereas the histology was con- + sidered to show pure talcosis.

A particle of talc from the case described is illustrated in Fig. 3, where the typical decoration pattern can be seen when thin slivers of the crystal are extracted on the replica, and shadowed ${ }^{\curvearrowright}$ with platinum. This effect on a particle of natural $\vec{\ominus}$ talc is also demonstrated in Figure 4. This same. decoration can also be observed on examination $\square$ of anthophyllite, often mistaken for talc, and used as such. The third patient was invalided out $\bar{\partial}$ of the army with a condition diagnosed as silicosis, and died at the age of 40. The necropsy $\underset{\perp}{\mathscr{Q}}$ revealed typical massive fibrosis. A histological $\underset{\vec{\circ}}{\overrightarrow{7}}$ diagnosis of a fibrosis caused by dust of unknown $\frac{}{3}$ constitution was made. Figures $5 \mathrm{a}, 5 \mathrm{~b}, 5 \mathrm{c}$ and $5 \mathrm{~d} J$ illustrate some of the large quantities of mica crystals observed in replicas from sections of lung tissue from this case. The planes of cleavage? are constant in all the photomicrographs studied. $\frac{5}{3}$ Difficulty was experienced in finding any particles using the low powers of the electron microscope $\frac{\rho}{3}$ and scanning had therefore to be carried out at $20 \times 10^{3}$. The amorphous nature of the crystal $\frac{}{5}$ surface, characteristic of mica, can be easily seen. $\frac{D}{O}$

Two patients with asbestosis were studied; the first, aged 46 when he died, presented with the $N$ usual symptoms of dyspnoea and productive $N$ cough. His industrial history began in 1931 when $\mathcal{C}^{-}$ he was employed in the crushing room of an $\frac{\omega}{\sigma}$ asbestos factory. In 1934 he was pensioned off with asbestosis.

Asbestos bodies were found in the sputum and $\stackrel{D}{\perp}$ at necropsy his lungs showed a marked pulmonary asbestosis. An electron micrograph of a mass of fibres found in the lung is shown in Figure 6. $\overrightarrow{\mathbb{D}}$ Comparison with electron micrographs of pure $\frac{\rho}{1}$ asbestos indicated that this mass of material was $\varrho$ made up of chrysotile fibres. No other type of asbestos was found in this tissue.

The second patient, aged 57 at the time of his death, also presented with dyspnoea and the usual productive cough and $x$-ray studies showed $\rightleftharpoons$ 


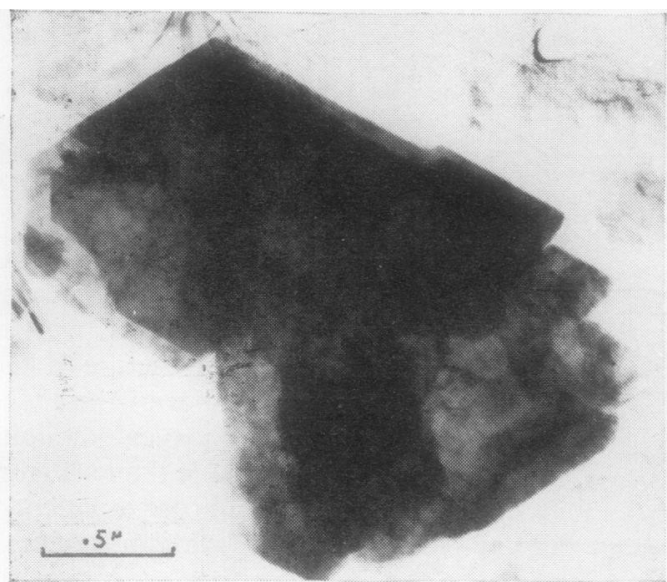

Fig. 2 Particle of kaolin-type crystals exhibiting typical $120^{\circ}$ fracture angle, extracted from lung tissue.

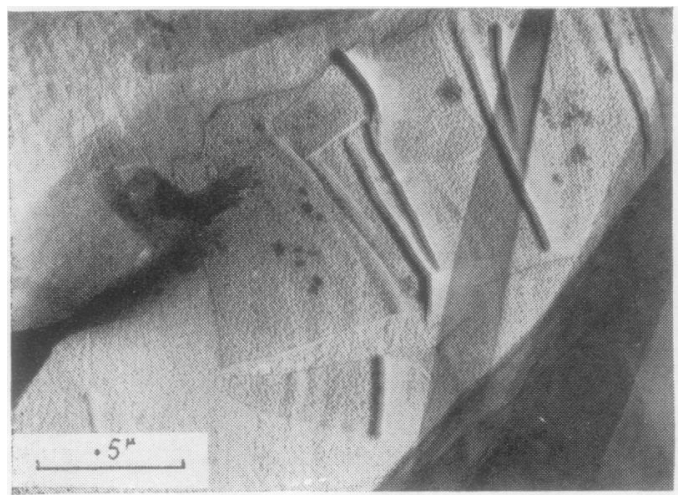

Fig. 4 Natural particle of talc.

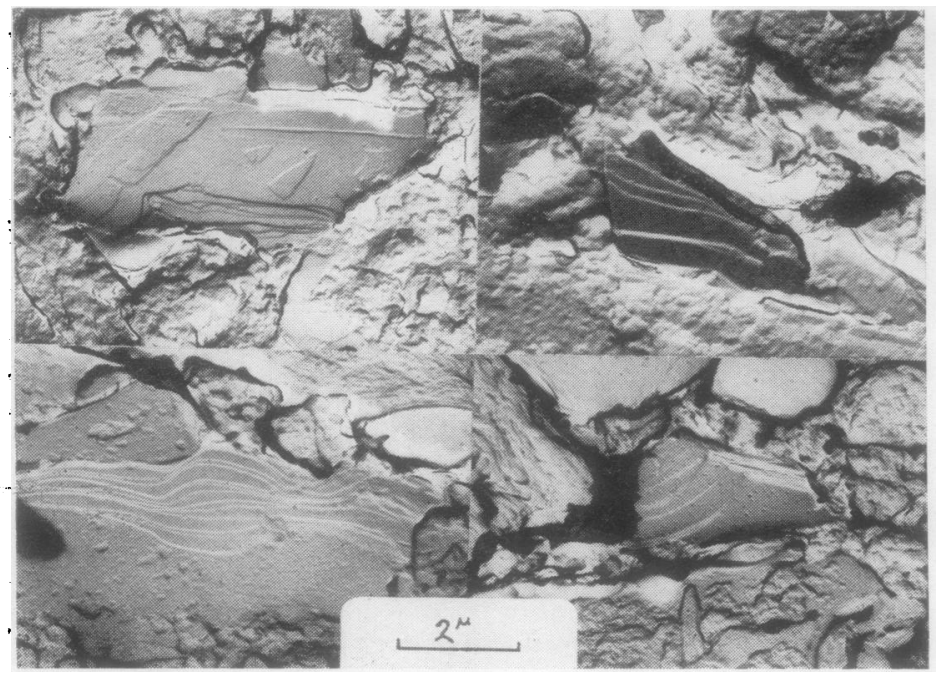

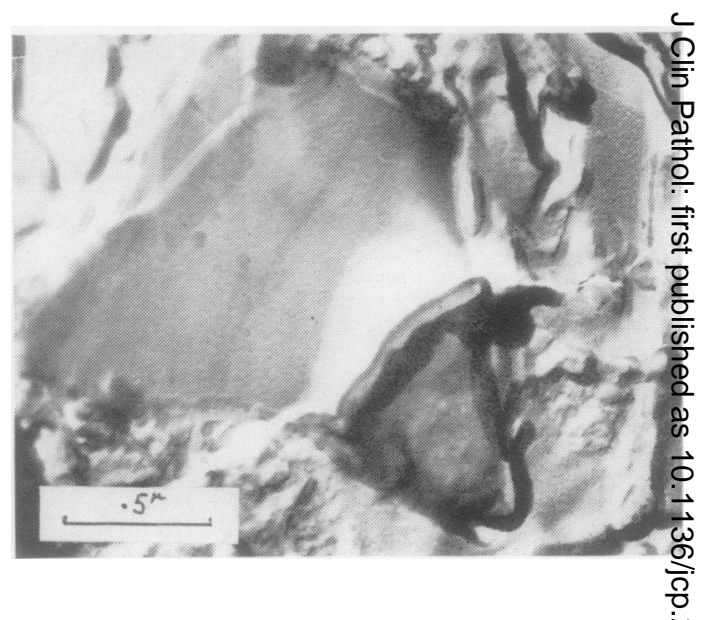

Fig. 3 Talc particle embedded in tissue demonstrating the typical decoration pattern obtained on this type of material when bombarded with platinum in vacuo.

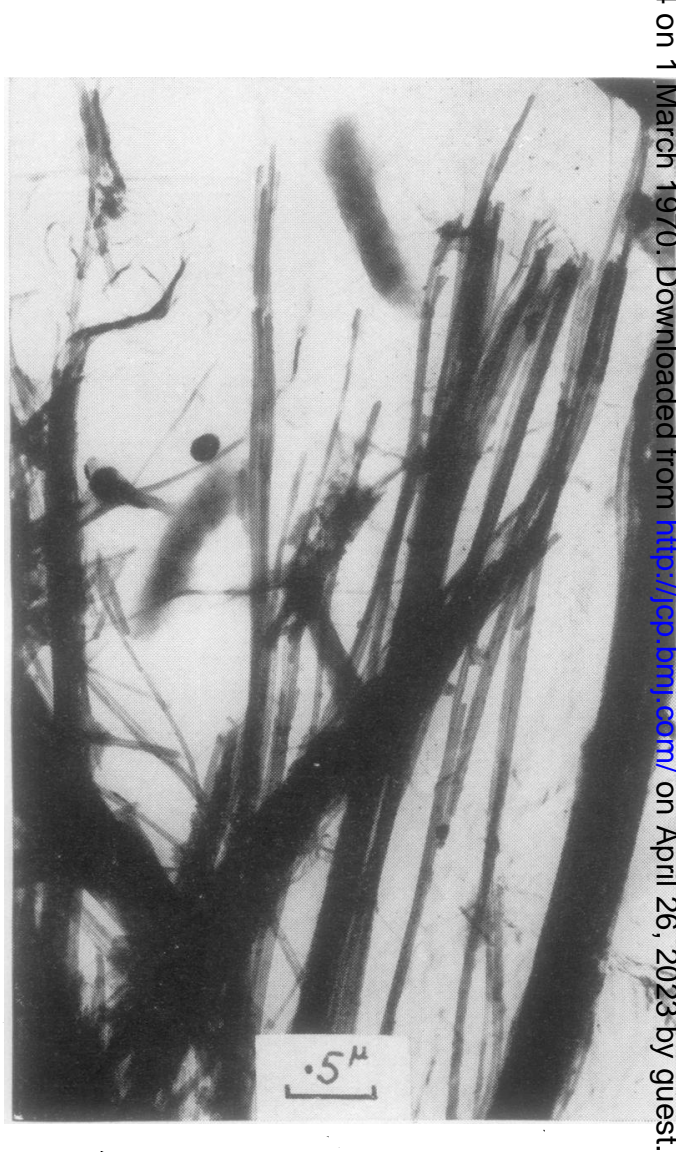

Fig. 6 Chrysotile asbestos fibres extracted from the $\frac{0}{\frac{0}{0}}$ lung tissue.

Fig. 5 Composite micrograph of lung sections containing mica particles embedded deep in the tissue. $\stackrel{\circ}{\circ}$

$\overrightarrow{3}$ 


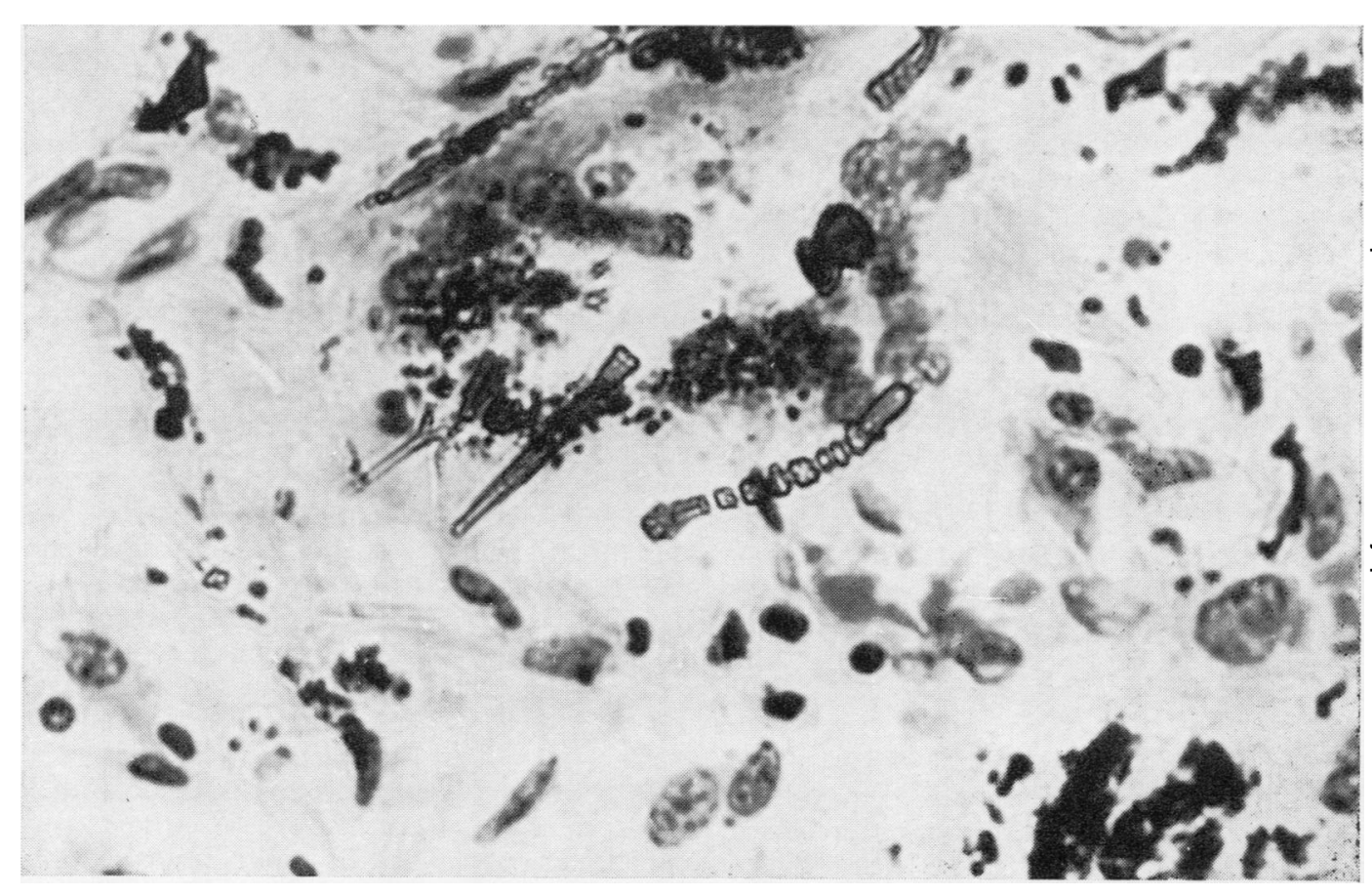

$\stackrel{1}{\prime \prime}$

$\underline{\Omega}$

\section{$\frac{1}{20}$}

Fig. 7 Optical micrograph of histology section showing asbestos bodies. No identification of the types of asbestos is possible at this level.

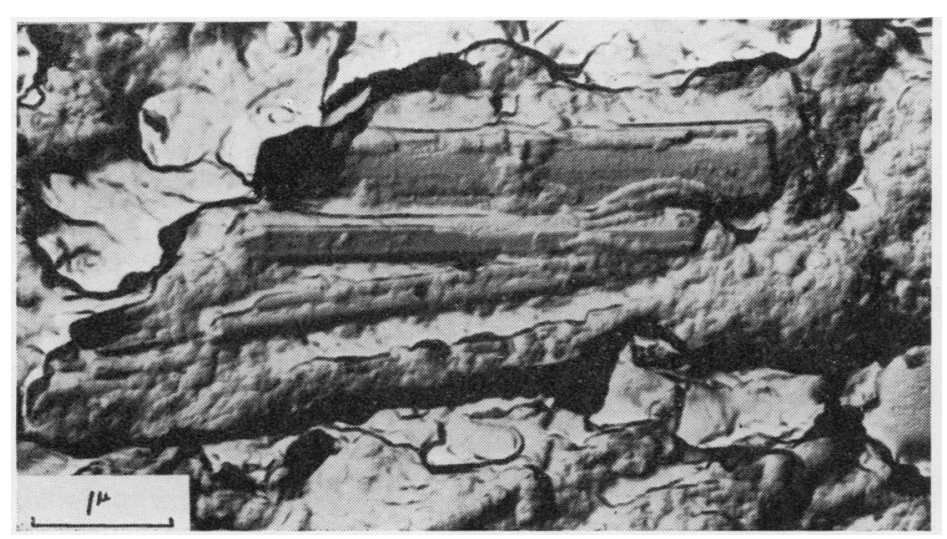

Fig. 8 Asbestos particles embedded in lung tissue. Collagen fibres can be seen between two of the particles. Note the $90^{\circ}$ fracture angle suggestive of amosite group.

Fig. 9 Replica of asbestos body, showing partial stripping of the ferritin granules from the fibre.

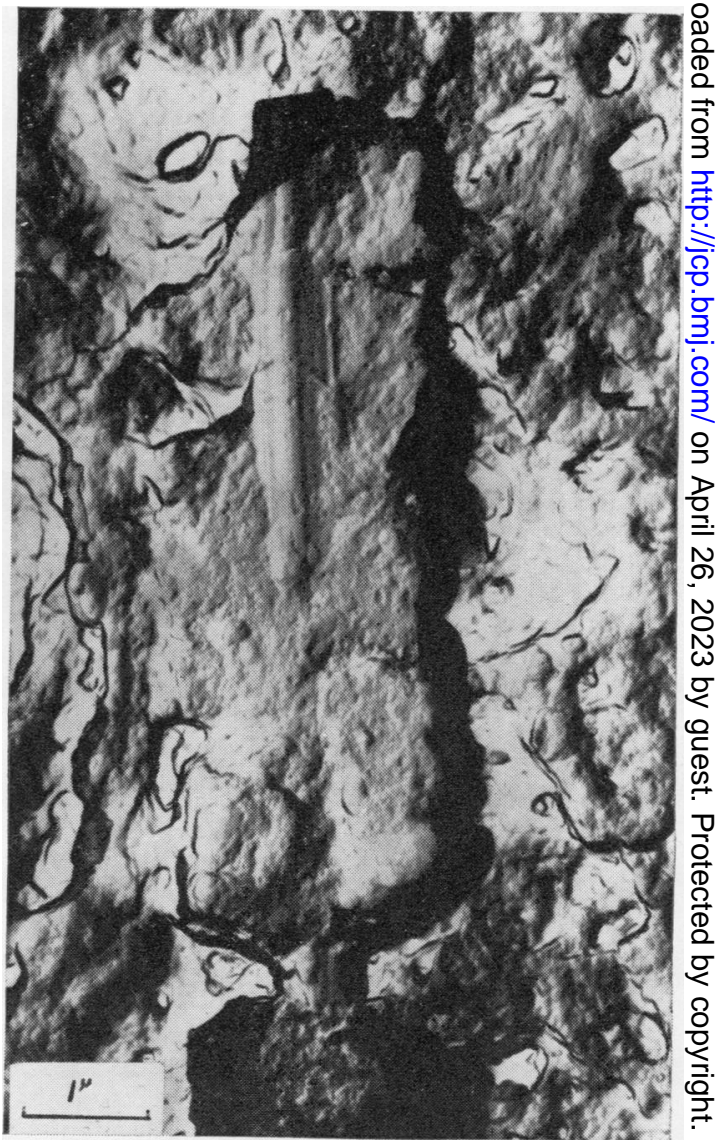


a thickened pleura. The whole of his working life of 30 years was spent with various types of asbestos. He started as a mattress maker, which involved the use of asbestos, from whence he moved to a shipyard where he used asbestos for the lagging of pipes in ships. At necropsy, a microscopic diagnosis of asbestosis was made. An optical micrograph of the lung tissue from this patient showing typical beaded asbestos bodies is illustrated in Figure 7. Electron micrographs of tissue from the same region confirmed the presence of askestos particles (Fig. 8), and the $90^{\circ}$ fracture angle of the fibres suggestive of the amosite group of asbestos, some surrounded by the characteristic beads of ferritin (Figure 9). This latter micrograph demonstrates the stripping of the ferritin bead, to expose the asbestos fibre, by the replication technique.

\section{Discussion}

The investigations reported in this communication provide a clear illustration of the use of a relatively simple replication technique for the study of mineral particles in pathological tissues. As well as the identification of the particles accumulated in the tissue, the technique enables the position of the particle in situ to be studied in relation to the surrounding tissue. This is seen in particular in Fig. 5, which shows the mica particles deeply embedded in the surrounding lung.

In these investigations on lung tissue from patients with pneumoconiosis, it was of interest to observe that in the first patient referred to the case was diagnosed as one of kaolinosis, and the mineral particles were easily seen with polarized light, the identity therefore being clear to the histologist. The electron microscope studies confirmed these findings. Similarly, in case 2, electron microscopy identified the talc particles within the lung tissue.

Case number 3 was of particular interest. Neither the industrial history nor the histology offered any conclusion on the aetiology of the fibrosis. Clinically the patient was considered to be suffering from silicosis (Gilson, 1960). The replication technique and electron microscopy indicated that particles responsible for the fibrosis were crystals of mica but the patient's history failed to provide evidence as to its source. Since no other particles were found, despite exhaustive investigation, it was assumed that mica is a factor in the causation of pneumoconiosis and this is probably supported by the fact that a number of cases with similar histology are described in Indian men who had worked in mica mines (Heimann, Moskowitz, Iyer, Gupta, and Mankiker, 1953).

The clinical diagnosis of asbestosis in the fourth and fifth cases referred to was confirmed by histology. The photomicrograph (Fig. 7) provided evidence for the presence of asbestos bodies but $\stackrel{\varrho}{=}$ was of little value in identifying the particle $\vec{J}$ causing the fibrosis. However, the electron microscopy studies revealed the value of the replication $\bar{\sigma}$ technique, and it was shown that the asbestos present was of the chrysotile group in the first $\overrightarrow{\vec{F}}$ case, and of the amosite or crocidolite group in $\overrightarrow{0}$ the second (Fig. 8 suggests the presence of fibres $\frac{C}{0}$ of amosite, since these tend to fracture at $90^{\circ}$, $\overline{\bar{c}}$ perpendicular to the plane or axis of the fibre). $\Phi$ Although it has been established for a number of years that these asbestos fibres are responsible for ${ }^{\infty}$ the fibrosis associated with asbestosis, these $\vec{\circ}$ studies confirmed the presence of the particles $\overrightarrow{\vec{\omega}}$ within the tissue.

The studies described also serve to indicate $\frac{\overline{0}}{0}$ that in the investigation of pathological specimens, in which mineral particles form one of the aetiological factors, the particles are often of dimensions $\stackrel{N}{-}$ too small for adequate identification by optical $\triangleright$ microscopy. The replication technique described 응 is of particular value in the identification of these particles and especially with its capacity to enable $z$ successive PVA stripping to be taken from the tissue samples, in order to find particles present $\stackrel{\sigma}{\partial}$ in relatively low density. The technique of ashing $\vec{\varphi}$ tissue samples before using the electron micro- $O$ scope does provide some indication of the particle density within the tissue and a means of adequate identification. Figure 10 shows asbestos bodies from ashed lung tissue, with the characteristic ferritin bodies on some fibres. These bodies are $\frac{\circ}{\Phi}$ usually the sole means of identifying asbestos by optical microscopy, but the ferritin bodies are difficult to differentiate from the similarly shaped bodies observed around elastin fibres (Gough, 1965). Figure 10 also illustrates the difficulty of resolving the fibres in optical systems, $\frac{8}{8}$ since many are $100 \mu$ long but $0.2 \mu$ wide. This $\dot{0}$ procedure, together with the replication technique $\frac{3}{.}$. for the study of the particle in situ, are extremely $\delta$ useful developments in the investigation of patients subject to dust exposure when expert study 은 is required for the elucidation of the causative factor.

It is to be expected that similar studies on the relationship between crocidolite in lung os tissue and the occurrence of mesotheliomas will $N$ provide similar results on the identification of the asbestos fibre associated with this neoplastic $\sigma$ condition, and studies along these lines have been 0 developed.

Of interest to note is the fact that this technique $\stackrel{\mathscr{D}}{+}$ can also be used in electron diffraction studies 0 when carbon alone is deposited on the PVA replicas and the crystals extracted examined for $\stackrel{\square}{\stackrel{D}{Q}}$ lattice parameters. Differentiation between amo- $\mathbb{\Phi}$ site and crocidolite has always been a particular $\bar{\sigma}$ problem. The use of the laser optical diffractometer may well be of value in the measurement of $O$ crystal parameters when used in association with this technique.

Also, in contrast to normal electron micro-? 


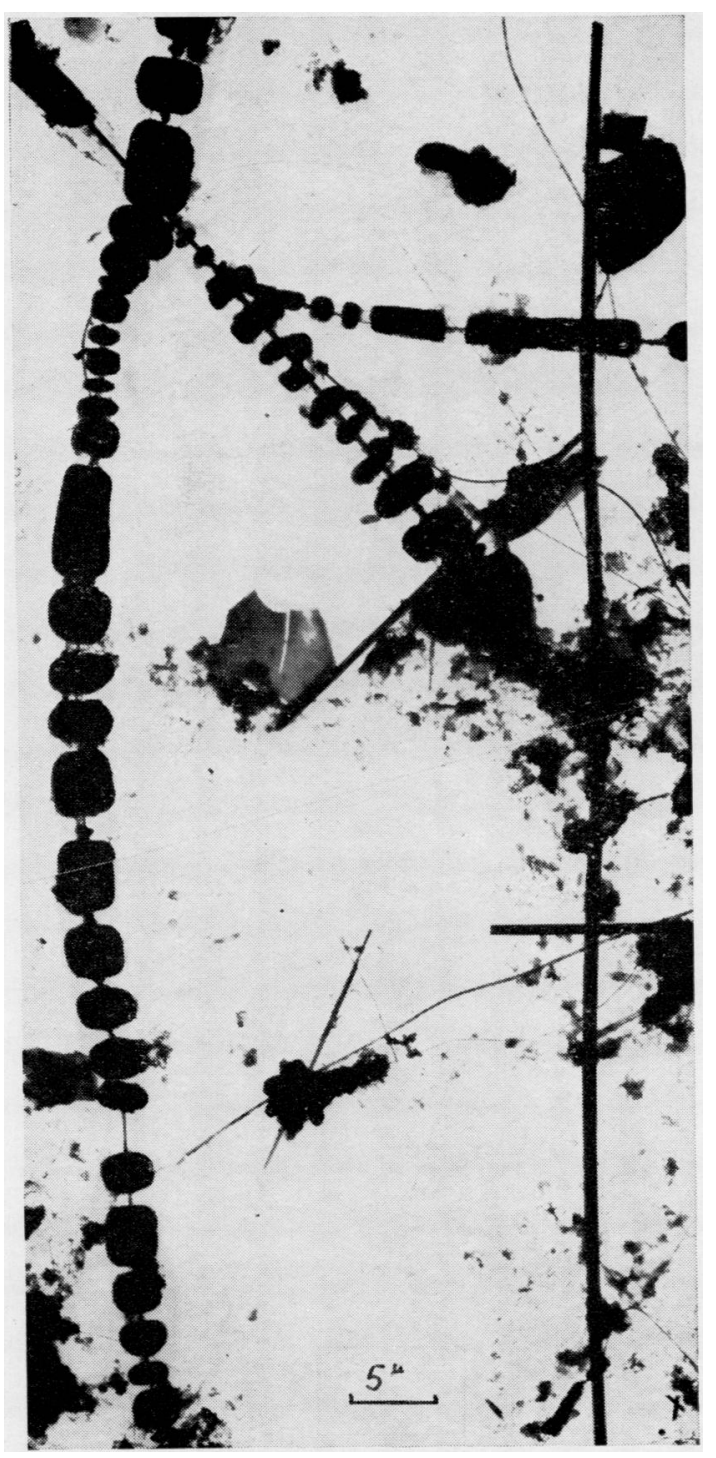

Fig. 10 Asbestos bodies obtained from ashed lung section. Note the length of fibre compared with its width, and the different types of decoration of the ferritin on the fibres. scopy procedure, the replication technique can be applied to the retrospective studies of tissue from patients, prepared as unstained paraffin sections, and stored in the record systems of pathology departments.

The authors wish to acknowledge the generous financial help of the Tenovus organization in Cardiff. They are also grateful for the encouragement and support of $\mathrm{Dr} \mathrm{K}$. Griffiths of the Tenovus Institute, the Welsh National School of Medicine, Cardiff. They also thank Mr D. E. Evans, Department of Geology, National Museum of Wales, for the minerals required for reference purposes.

\section{References}

Gilson, J. C. (1960). Industrial pulmonary disease. In Modern Trends in Occupational Health, edited by R. S. F. Schilling. Butterworth, London.

Gough, J. (1965). Differential diagnosis in the pathology of asbestosis. Ann. N.Y. Acad. Sci., 132, 368-372.

Heimann, H., Moskowitz, S., Iyer, C. R. H., Gupta, M. N., and Mankiker, N. S. (1953). Silicosis in mica mining in Bihar, India. A.M.A. Arch. industr. Hyg., 8, 420-435.

Henderson, W. J. (1969). A simple replication technique for the study of biological tissues by electron microscopy. $J$. Microsc., 89, 369-372. 